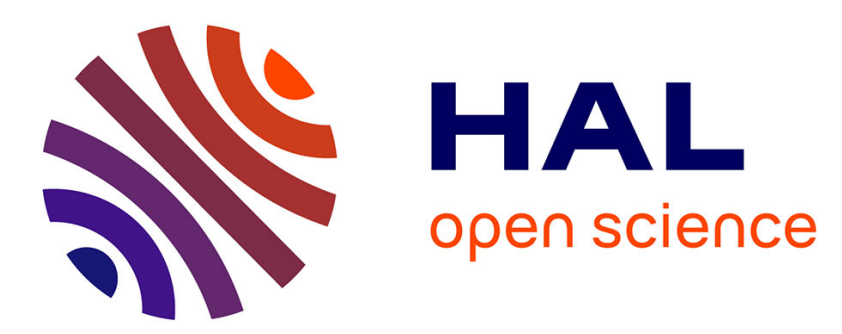

\title{
Extraction of Foetal Contribution to ECG Recordings Using Cyclostationarity-Based Source Separation Method
}

\author{
Michel Haritopoulos, Cécile Capdessus, Asoke K. Nandi
}

\section{To cite this version:}

Michel Haritopoulos, Cécile Capdessus, Asoke K. Nandi. Extraction of Foetal Contribution to ECG Recordings Using Cyclostationarity-Based Source Separation Method. Ninth International Conference on Latent Variable Analysis and Signal Separation, Sep 2010, St. Malo, France. pp.588-595, 10.1007/978-3-642-15995-4_73. hal-00653536

\section{HAL Id: hal-00653536 https://hal.science/hal-00653536}

Submitted on 19 Dec 2011

HAL is a multi-disciplinary open access archive for the deposit and dissemination of scientific research documents, whether they are published or not. The documents may come from teaching and research institutions in France or abroad, or from public or private research centers.
L'archive ouverte pluridisciplinaire HAL, est destinée au dépôt et à la diffusion de documents scientifiques de niveau recherche, publiés ou non, émanant des établissements d'enseignement et de recherche français ou étrangers, des laboratoires publics ou privés. 


\title{
Extraction of Foetal Contribution to ECG Recordings Using Cyclostationarity-Based Source Separation Method
}

\author{
Michel Haritopoulos ${ }^{1}$, Cécile Capdessus ${ }^{1}$, and Asoke K. Nandi ${ }^{2}$ \\ 1 Institut PRISME \\ 21, rue de Loigny la Bataille, 28000, Chartres \\ \{Michel.Haritopoulos, Cecile.Capdessus\}@univ-orleans.fr, \\ http://www . univ-orleans.fr/prisme/ \\ 2 Signal Processing and Communications group, Department of Electrical \\ Engineering and Electronics \\ The University of Liverpool, Brownlow Hill, Liverpool L69 3GJ, U.K.
}

A. Nandi@liverpool.ac.uk

\begin{abstract}
In this paper we propose a cyclostationary approach to the problem of the foetal electrocardiogram (FECG) extraction from a set of cutaneous potential recordings of an expectant mother. We adopted a semi-blind source separation (BSS) method for which the only necessary prior knowledge is that of the fundamental cyclic frequency of the cyclostationary process to estimate. The estimated cyclostationary FECG source of interest is found to be free from any interferences with the mother's ECG (MECG) signal. Experimental results and perspectives for future research conclude this paper.
\end{abstract}

Key words: Cyclostationarity, Semi-Blind Source Extraction, Foetal Electrocardiogram

\section{Introduction}

Electrocardiograms (ECG) are very often used as diagnostic tools for heart monitoring. In the case of pregnant women, the recorded ECGs contain information concerning both mother's and foetus' condition. Early diagnosis in the case of the mother's ECGs (MECG) can be helpful for medical assessment of possible diseases, while in the foetus case (FECG), any prenatal information that can be provided may be of great importance to prevent complications. ECGs acquired with non-invasive techniques do not give direct access to FECGs, because those are hidden by the maternal ECGs of higher amplitude and contaminated by various sources of disturbances (biological or other).

It is shown in the literature ([1], [2], [3], [4]), that this problem can be formulated in blind source separation (BSS) terms. It consists of separating or extracting one or more signals of interest from a set of observations which contain a linear or non-linear mixing of the unknown source signals that one wants to estimate. 
In the case of ECG signals, $m$ electrodes are placed at several key locations on the mother's body and the observations carried out by these electrodes are modelled in terms of BSS as a vector $\mathbf{x}$ assumed to be a linear mixture of the unknown source vector $\mathbf{s}$ :

$$
\mathbf{x}(t)=A \mathbf{s}(t)
$$

where $\mathbf{s}(t)$ are the $n$ unknown sources $\left[s_{1}(t), s_{2}(t), \ldots, s_{n}(t)\right]^{\dagger} \in \mathbb{R}^{n \times 1}, \mathbf{x}(t)$ outputs the $m$ linearly mixed observations $\left[x_{1}(t), x_{2}(t), \ldots, x_{m}(t)\right]^{\dagger} \in \mathbb{R}^{m \times 1}, A$ stands for the $m \times n$ mixing matrix and $\dagger$ denotes the transpose operator. Equation (1) is the well known noiseless linear instantaneous model of the BSS problem.

Various approaches have been used to solve the FECG extraction problem by using BSS techniques. One can find in the literature second-order statistics (SOS) based BSS methods [5] or adaptive noise cancelling methods compared with higher-order statistics (HOS) based BSS techniques [6]. Sparse representations [7] as well as wavelet based ICA [9] applied to this particular problem have also been reported.

Here we choose to apply an algorithm that allows to extract one specific cyclostationary source whose cyclic frequency is a priori known. A preliminary study of the data indicates that the observations exhibit cyclostationarity at the frequency of the foetal heartbeat rate, which is estimated and then used for the extraction.

\section{Methods}

\subsection{Extraction Principle}

The extraction method was previously presented in [10]. It aims at extracting a cyclostationary source of known cyclic frequency $\alpha_{0}$ from a set of observations. We thus look for a $1 \times m$ extraction vector $B$ such that:

$$
z(t)=B \mathbf{x}(t)
$$

is an estimate of the cyclostationary source. Hypotheses are as follows:

The observations are instantaneous and additive mixtures of the sources.

$\square$ There are at least as many sensors as sources.

The sources are supposed to be zero mean and uncorrelated to each other.

$\square$ The source to be extracted is cyclostationary at a frequency $\alpha_{0}$ that is $a$ priori known, i.e. it can either be measured or computed.

$\square$ The other sources can be either stationary or cyclostionary, provided that none of them is cyclostationary at the same frequency $\alpha_{0}$. 
The extraction is performed by minimising over $B$ a criterion $C(B)$ based on second-order statistics of the observations. Let us denote by $R_{z}(t, 0)$ the autocorrelation function of the estimate $z(t)$ at zero time-lag. Then:

$$
C(B)=\left|\frac{R_{z}(0)}{R_{z}^{\alpha_{0}}(0)}\right|
$$

where $R_{z}(0)$ and $R_{z}^{\alpha_{0}}(0)$ are the coefficients of the Fourier series decomposition of $R_{z}(t, 0)$ at respective frequencies 0 and $\alpha_{0}$.

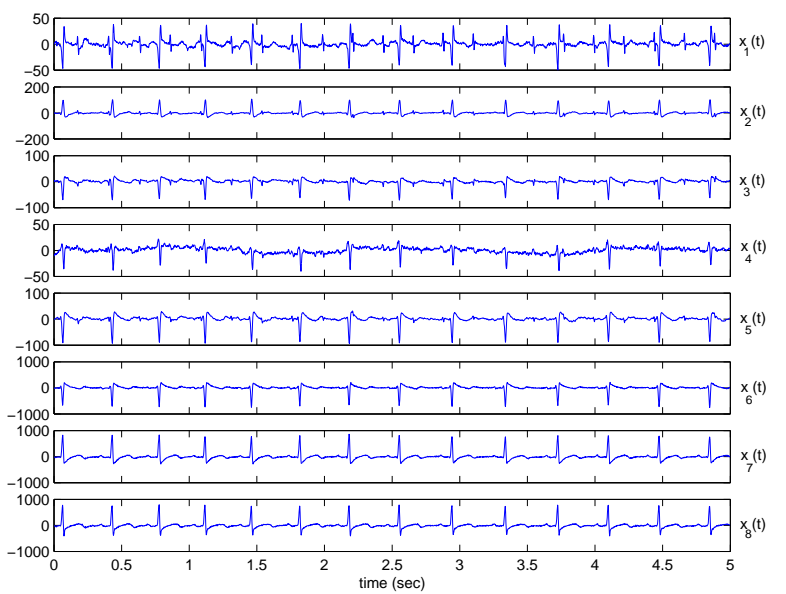

Fig. 1. Eight skin electrodes recordings form a pregnant woman

\subsection{Algorithm}

Let us denote by $\widehat{R}_{\mathbf{x}}(0)=\left\langle\mathbf{x}(t) \mathbf{x}^{\dagger}(t)\right\rangle_{\theta}$ and $\widehat{R}_{\mathbf{x}}^{\alpha_{0}}(0)=\left\langle\mathbf{x}(t) \mathbf{x}^{\dagger}(t) e^{-2 \pi j \alpha_{0} t}\right\rangle_{\theta}$ the estimates of the covariance matrix of the observations and their cyclic covariance matrix at frequency $\alpha_{0}$, respectively. The $\langle.\rangle_{\theta}$ operator stands for the temporal averaging over $\theta$ seconds. The criterion is estimated from the observations as follows:

$$
\widehat{C}(B)=\left|\frac{B \widehat{R}_{\mathbf{X}}(0) B^{\dagger}}{B \widehat{R}_{\mathbf{X}}^{\alpha_{0}}(0) B^{\dagger}}\right| .
$$

It has been shown in [10] that minimising eq. (4) over $B$ leads to the extraction of the corresponding cyclostationary component.

\section{Methodology and Results}

The proposed algorithm is very well adapted to the problem of FECG extraction as shown below. We apply it to a commonly used dataset in relation to FECG extraction research [11]. 


\subsection{Experimental Dataset}

The dataset we used is illustrated in Fig. 1. These are eight-channel cutaneous potential recordings from eight skin electrodes placed at different positions of an expectant mother's body. The first five observations denoted $\left[x_{1}(t), \ldots, x_{5}(t)\right]$ were recorded from the abdominal area, while the last three $\left[x_{6}(t), \ldots, x_{8}(t)\right]$ correspond to electrodes placed at the mother's thoracic region. All signals were sampled at frequency $f_{e}=500 \mathrm{~Hz}$ during $5 \mathrm{~s}$, so that the observations vector $\mathbf{x}(t)$ is $N=2500$ samples long. The foetal heartbeat component can be perceived together with MECG contribution and noise in the first $x_{1-5}(t)$ observations subset, while in the second one $x_{6-8}(t)$, the FECG contributions are barely visible because of the much longer distance between the foetus and the electrodes located on the mother's chest.

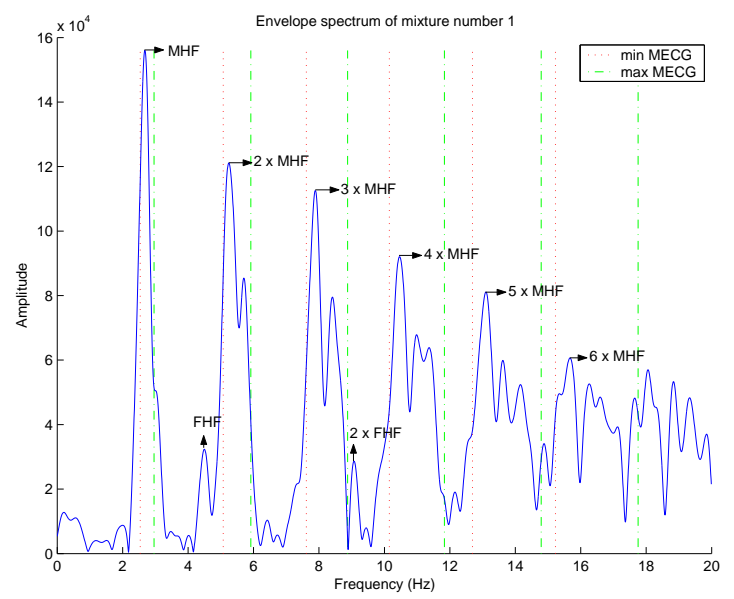

Fig. 2. Mother's (MHF) and foetus' (FHF) heartbeat frequencies and their harmonics. Theoretical evolution of the MHF within the $k \times[2.538,2.958] \mathrm{Hz}$ values range $(k=1, . ., 6)$ drawn in dotted and dash-dotted lines (minimum and maximum value, respectively).

We performed envelope spectrum analysis of the observations, i.e. the Fourier transform of the squared signals [12]. All eight channels give substantially the same envelope spectra; but as on the temporal ECG representations, it is at the first channel that the foetal heartbeat rate can be distinguished clearly. Fig. 2 is a zoom of the first mixture $x_{1}(t)$ vector's envelope spectrum; mother's heartbeat frequency (MHF) and its harmonic components $k \times M H F, k \in[1,6]$ are dominating the whole spectrum. The frequency intervals [ $\min M E C G, \max M E C G$ ] plotted with dotted and dash-dotted lines, respectively, correspond to the [2.538, 2.958] $\mathrm{Hz}$ frequency range values, within which mother's heartbeats vary. As expected, MHF lies in this interval. 
Foetus' heartbeat frequency (FHF) frequency and its second harmonic also appear in this figure. But, the widening of the MHF harmonics at higher frequencies, makes it difficult to point out with certainty which spectral line corresponds to a FHF harmonic. The presence of spectral lines in the envelope spectrum supports the thesis of second order cyclostationarity characterizing the FECG component, with a fundamental cyclic frequency $\alpha_{0}$ equal to $4.49 \mathrm{~Hz}$, i.e. the value of the FHF.

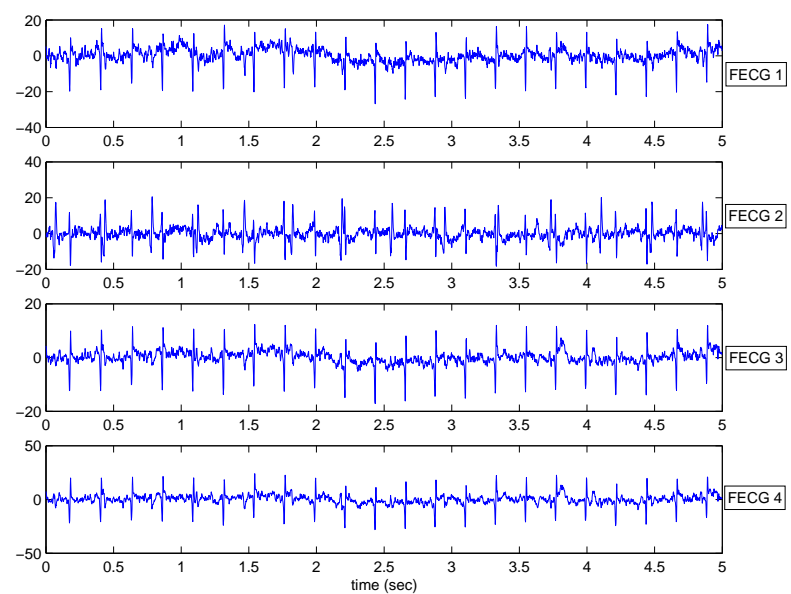

Fig. 3. From the top: four FECG estimations provided by the proposed algorithm with input mixture vectors $\mathbf{x}_{1}(t), \ldots, \mathbf{x}_{4}(t)$, respectively.

\section{$3.2 \quad$ Experiments and Results}

We carried out experiments by applying our algorithm to different sets of observations. We describe, hereafter, the results obtained by using four different observations vectors. The first mixture vector $\mathbf{x}_{1}(t)$ is composed of observations $\left[x_{1}(t), x_{2}(t), x_{3}(t)\right]^{\dagger}$, the second one $\mathbf{x}_{2}(t)=\left[x_{1}(t), x_{2}(t), x_{5}(t)\right]^{\dagger}$, the third one $\mathbf{x}_{3}(t)$ is $\left[x_{1}(t), x_{3}(t), x_{5}(t)\right]^{\dagger}$ and the last one is made of four observations vectors $\mathbf{x}_{4}(t)=\left[x_{1}(t), x_{2}(t), x_{3}(t), x_{5}\right]^{\dagger}$ (see Fig. 1).

Note, that, we did not include into our mixtures vectors recorded data from channel number 4, to which FECG contribution is very low. Furthermore, baseline wander is observed in this channel as this is the case in many other ECG datasets. This introduces amplitude variations to all separated FECG contributions after application of a BSS algorithm [7]. One can find in the literature (e.g., [8]) preprocessing methods for baseline wander removal and bandpass filtering of ECG recordings. After running the new cyclostationary criterion based BSS algorithm [10], with fundamental FECG cyclic frequency $\alpha_{0}=4.49 \mathrm{~Hz}$ as estimated from the first channel's envelope spectrum, we obtain for each mixture 
vector $\mathbf{x}_{i}(t), i \in[1,4]$ an estimate of the foetal electrocardiogram signal. Extraction results are shown in Fig. 3. The extracted FECG that exhibits both very little baseline wandering and the foetus heartbeats, is the last estimate, i.e. the one that used information from 4 channels for minimising criterion of eq. (4).

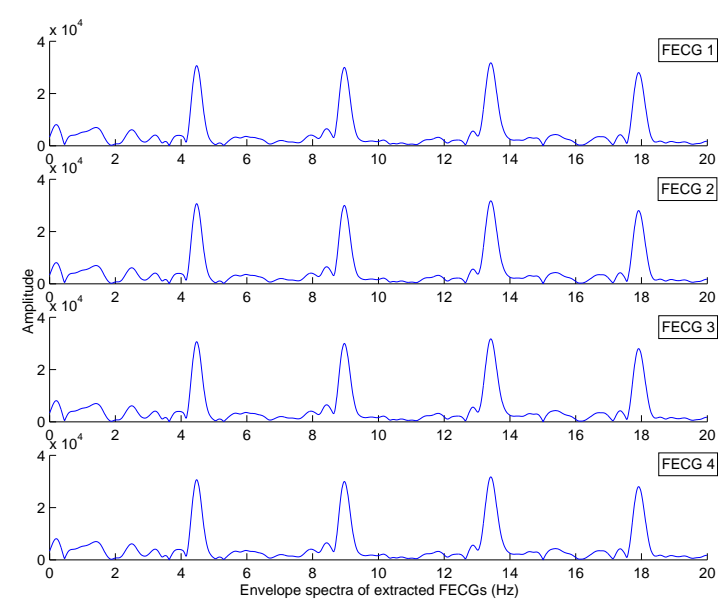

Fig. 4. From top to bottom: zoom to the envelope spectra of the extracted FECGs by using 4 different mixture vectors.

Fig. 4 shows the envelope spectra of the extracted FECG signals after application of the proposed estimation criterion algorithm to the mixture vectors $\mathbf{x}_{i}(t), i \in[1,4]$ (from top to bottom). In order to compare these results with the envelope spectra of Fig. 2, we plotted a zoom for each extracted FECG signature. It is worth pointing out that our algorithm works well for all different combinations of the mixture vector components, as well as for a different observations number $m$. Indeed, one can see in this figure that, the FHF fundamental frequency and its next three harmonics are not contaminated by any MHF spectral lines. Experiments have been carried out with all eight channels ECG recordings as input mixing vector to our extraction algorithm. A study of the FECG signal spectrum extracted after application of our algorithm to the whole ECG recordings set, confirms the existence of the first MHF harmonic, while no MHF components can be found in the spectrum computed from the extracted FECG using $x_{4}(t)$. This could be justified by assuming that recordings from the mother's thoracic region bring very few new information concerning the foetus' heartbeats.

\subsection{Robustness to Foetus's Cyclic Frequency}

The only prior knowledge required for our estimation method to work, is that of the foetus's fundamental cyclic frequency value $\alpha_{0}$. As explained in section 3.2, 
we used a value of $4.49 \mathrm{~Hz}$ for $\alpha_{0}$ as it is estimated from the envelope spectrum of the first ECG channel recordings. Deviations from this value can be detected depending which channel's envelope spectrum the estimation is based on. Thus, we run some experiments with the previously described $x_{4}(t)$ mixing vector. At each run, we applied the proposed cyclostationary extraction method to $x_{4}(t)$ with a different $\alpha_{0}$ value and we computed a separation quality criterion for the corresponding estimated FECG signal. This is a periodicity measure (PM $\in[0,100])$ based on second-order statistical properties and introduced in [8]; it is computed at the mean mother's heartbeat rate without taking into account its variability:

$$
P M=\frac{\left|E\left\{x(t) x\left(t+\tau_{\mu}\right)\right\}\right|}{\sqrt{E\left\{x(t)^{2}\right\} E\left\{x\left(t+\tau_{\mu}\right)^{2}\right\}}} * 100 \%,
$$

where $\tau_{\mu}$ is the mean mother's heartbeat period. If the extracted FECG is clear of MHF components, then PM should be $0 \%$.

Computed PMs for $\alpha_{0}$ values over the frequency range of interest, i.e., from $4.4 \mathrm{~Hz}$ to $4.6 \mathrm{~Hz}$ by steps of $0.01 \mathrm{~Hz}$, take values $<0.5 \%$. PM values computed for every single ECG channel lie within $[17,29] \%$. This means that, even by introducing small variations on the estimated $\alpha_{0}$ value, the extraction algorithm still provides estimate FECG signals with about three times better quality in therms of PM with respect to the best ECG channel. Therefore, for a given ECG channel, the estimation of the foetus fundamental cyclic frequency from its envelope spectrum seems to be robust for small deviations from the true value of $\alpha_{0}$.

\section{Discussions and Conclusions}

We have introduced in this paper a novel application of our cyclostationaritybased BSS algorithm to the problem of foetus heartbeat signals extraction from a pregnant's woman ECG recordings. We pointed out the main lines of the estimation principle and then successfully applied it to real ECG signals.

To use the proposed method, there is no need to prewhiten (by the principal component analysis, for example) the sensor signals; centering the observations (zero mean) and decorrelation assumption are sufficient. We just have to know/estimate the fundamental cyclic frequency of the cyclostationary process (i.e. the FECG signal, in this study) to extract from the mixtures. It then estimates the source signal of interest. In the case presented in this paper, since the cyclostationary frequency chosen for the extraction was that of the FECG signal, the estimated source fits the heartbeats of the foetus. The indeterminacies inherent to BSS methods, i.e. the sign and the amplitude of the separated source signal, still remain in the proposed method. But post processing of the extracted FECG signal may be used to compute its contribution to each sensor.

Further experiments are under progress in order to be able to compare our algorithm's performance with that of similar work reported in the literature; its robustness to the foetus' cyclic frequency is also studied for different ECG 
channels and different number of mixtures. Taking into account the presence of baseline wander in some sensors, extracting the mothers's heartbeat signals and the characteristic waveforms associated with each heartbeat are of great interest in order to perform a robust monitoring of the foetus well-being.

\section{References}

1. Lathauwer, L.D., Moor, B.C., Vandewalle, J.: Foetal Electrocardiogram Exctraction by Blind Source Subspace Separation. In: IEEE Trans. Biomed. Eng., vol. 47, no. 5, pp. 567-572 (2000)

2. Zarzoso, Z., Nandi, A.K., Bacharakis, E.: Maternal and Foetal ECG Separation Using Blind Source Separation Methods. In: IMA Journal of Mathematics Applied in Medicine \& Biology, vol. 14, no. 3, pp. 207-225 (1997)

3. Bacharakis, E., Nandi, A.K., Zarzoso, V.: Foetal ECG Extraction Using Blind Source Separation Methods. In: Proceedings of EUSIPCO-96, eds. G Ramponi, G L Sicuranza, S Carrato, and S Marsi, Edizioni Lint Trieste, Italy, vol. 1, pp. 395-398 (1996)

4. Bacharakis, E., Nandi, A.K., Zarzoso, V.: Foetal ECG Separation from Surface Measurements. In: IMA Conference on Mathematics in Medicine and Biology, Oxford, UK (1996)

5. Jafari, M.G., Wang, W., Chambers, J.A., Hoya, T., Cichocki, A.: Sequential Blind Source Separation Based Exclusively on Second-Order Statistics Developed for a Class of Periodic Signals. In: IEEE Transaction on Signal Processing, vol. 54, no. 3, pp. 1028-1040 (2006)

6. Zarzoso, V., Nandi, A.K.: Noninvasive Foetal Electrocardiogram Extraction: Blind Separation Versus Adaptive Noise Cancellation. In: IEEE Transactions on Biomedical Engineering, vol. 48, no. 1, pp. 12-18 (2001)

7. T., Blumensath T., Davies, M.E.: Blind Separation of Maternal and Foetal ECG Recordings using Adaptive Sparse Representations. In: Proceedings of ICA Research Network International Workshop 2006, In: A K Nandi and X Zhu (eds.), Liverpool, UK (2006)

8. Sameni R., Jutten C., Shamsollahi M. B.: A Deflation Procedure for Subspace Decomposition. In: IEEE Transactions on Signal Processing, vol. 58, no. 4, pp. 2363$2374(2010)$.

9. Azzerboni, D., La Foresta, F., Mammone, N., Morabito, F.C.: A New Approach Based on Wavelet-ICA Algorithms for Foetal Electrocardiogram Extraction. In: Proceedings ESSAN'2005, d-side publi., Bruges, Belgium (2005)

10. Capdessus, C., Nandi, A.K., Bouguerriou, N.: Source Extraction Algorithm Based on Cyclic Properties. In: Proceedings of ICA Research Network International Workshop 2008, Liverpool, UK, pp. 36-39 (2008)

11. (1999, May) DaISy; Database for the Identification of Systems. ESAT/SISTA, K. U. Leuven, Belgium. [Online], Available: http://homes.esat.kuleuven.be/ smc/ daisy/

12. Randall, R.B., Antoni, J., Chobsaard, S.: The Relationship between Spectral Correlation and Envelope Analysis for Cyclostationary Machine Signals. Application to Ball Bearing Diagnostics. In: Mechanical Systems and System Processing, vol. 15, no. 5, pp. 945-962 (2001) 